\title{
THE
}

3-14-1994

\section{Anomalous Spin Dynamics and Relaxation in Fermi Liquids}

Alexander E. Meyerovich

University of Rhode Island, sfo101@uri.edu

K. A. Musaelian

University of Rhode Island

Follow this and additional works at: https://digitalcommons.uri.edu/phys_facpubs

Terms of Use

All rights reserved under copyright.

\section{Citation/Publisher Attribution}

Meyerovich, A. E., \& Musaelian, K. A. (1994). Anomalous Spin Dynamics and Relaxation in Fermi Liquids. Phys.Rev.Lett., 72, 1710-1713. doi: 10.1103/PhysRevLett.72.1710

Available at: http://dx.doi.org/10.1103/PhysRevLett.72.1710

This Article is brought to you for free and open access by the Physics at DigitalCommons@URI. It has been accepted for inclusion in Physics Faculty Publications by an authorized administrator of DigitalCommons@URI. For more information, please contact digitalcommons-group@uri.edu. 


\title{
Anomalous Spin Dynamics and Relaxation in Fermi Liquids
}

\author{
A. E. Meyerovich and K. A. Musaelian* \\ Department of Physics, University of Rhode Island, Kingston, Rhode Island 0288I
}

(Received 23 November 1993)

\begin{abstract}
We explain the anomalous temperature dependence of spin diffusion in liquid ${ }^{3} \mathrm{He} \uparrow$ and ${ }^{3} \mathrm{He} \uparrow-{ }^{4} \mathrm{He}$ mixtures. The anomaly is an experimental manifestation of a unique zero-temperature attenuation in the Fermi liquid theory. We extended our microscopic theory of spin dynamics in spin-polarized Fermi liquids to finite temperatures. The zero-temperature attenuation changes the behavior of the spectrum near the singular point. The data indicate that the superfluid transition temperature for ${ }^{3} \mathrm{He}$ in ${ }^{3} \mathrm{He}-{ }^{4} \mathrm{He}$ mixtures is much lower than the current estimates.
\end{abstract}

PACS numbers: $67.65 .+\mathrm{z}, 67.60 . \mathrm{Fp}, 72.15 . \mathrm{Nj}$

Experiments [1,2] in ${ }^{3} \mathrm{He} \uparrow$ and ${ }^{3} \mathrm{He} \uparrow-{ }^{4} \mathrm{He}$ mixtures demonstrated that the transverse spin diffusion coefficient $D_{\perp}$ and the relaxation time $\tau_{\perp}$ do not increase with decreasing temperature as $1 / T^{2}$, but saturate below some temperature $T_{a}$ :

$$
D_{\perp}=\frac{D_{\perp 0} T_{a}^{2}}{T_{a}^{2}+T^{2}}, \quad \tau_{\perp}=\frac{\tau_{\perp 0} T_{a}^{2}}{T_{a}^{2}+T^{2}} .
$$

Experimental values of $T_{a}$ are $16.4 \pm 2.2 \mathrm{mK}$ for liquid ${ }^{3} \mathrm{He} \uparrow$, and $14 \mathrm{mK}$ for ${ }^{3} \mathrm{He} \uparrow-{ }^{4} \mathrm{He}$ mixtures with $3.8 \%{ }^{3} \mathrm{He}$.

The deviation from the standard $1 / T^{2}$ dependence of transport coefficients in Fermi liquids can be explained only by a unique zero-temperature attenuation in transverse spin dynamics of spin-polarized Fermi liquids which was predicted earlier by one of the authors [3]. The idea is based on symmetry and phase space arguments. The transverse spin dynamics (the dynamics of components of magnetization that are perpendicular to the external magnetic field) is the motion of off-diagonal (mixed) spin states of particles which, in contrast to pure spin-up and spin-down states, do not fill any Fermi sphere. The conservation laws do not restrict collisions of particles in these mixed states to a thin belt near the Fermi surface, and the phase space for such collisions is much larger than for the pure spin-up and spin-down states. Thus the attenuation of the mixed states is much larger than for the pure states, does not contain a small Fermi liquid factor $\left(T / T_{0}\right)^{2}$, and should not necessarily go to zero at $T \rightarrow 0$. The zero-temperature attenuation is not a relaxation in a narrow sense, but is a reflection of decay of single-particle states away from the Fermi surface.

Later, the existence of the zero-temperature attenuation has been confirmed by transport calculations [4] for dilute Fermi gases. An extrapolation to higher densities gives a much lower transition temperature $T_{a}$ (1) than the experimental values [1,2]. This is not unexpected since the calculations [4] are based on a transport equation which is valid only for systems with very low densities.

The zero-temperature attenuation lies beyond the standard Landau-Silin-Leggett (LSL) theory of spin dynamics in spin-polarized Fermi liquids [5]. In essence, the
LSL description is a zero polarization limit of the "exact" theory. The problems in transverse spin dynamics of highly polarized Fermi systems have been known for several decades (mostly unpublished; see a brief summary in [6]). However, the success of the LSL theory of weakly polarized Fermi liquids masked a lack of understanding of spin dynamics in highly polarized systems.

Recently we have derived exact microscopic equations for spin dynamics in polarized Fermi liquids at $T=0$ via the self-energy functions of pure states and the mixed spin component of the irreducible vertex [7]. The only assumption is that the Green's functions for pure spin states have a standard Fermi liquid singularity. The results at $T=0$ reveal the microscopic origins of the zerotemperature attenuation, but cannot be compared with the finite-temperature data $[1,2]$. Besides, the equations contain microscopic parameters which are unknown for ${ }^{3} \mathrm{He}$. The results [7] become very simple (similar to [4]) at low density, but this does not help for dense systems.

Below we will lift these limitations. At low spin polarization $(H \sim 9 \mathrm{~T}$ in $[1,2]$ corresponds to the Zeeman energy $2 \beta H \sim 14 \mathrm{mK})$, the exact equations [7] can be simplified and extended to finite temperatures. The results provide a good quantitative description of the experiments $[1,2]$.

Since in the zero polarization limit the equations [7] reproduce the standard LSL theory, it is sufficient to leave the real parts of the vertex and the mass operators in the LSL form, and add the lowest in polarization imaginary corrections to the vertex and self-energy. We will not give the details; we will only outline the procedure and analyze the results.

Even the low-frequency transverse spin dynamics involves the single-particle states between the Fermi spheres for spin ups and spin downs [6]. The mixed spin states oscillate between these Fermi surfaces, $|\mathbf{p}|=p_{1, l}$, and the surfaces $|\mathbf{p} \mp \mathbf{k} / 2|=\left|\mathbf{p}_{1,1} \pm \mathbf{k} / 2\right|$. The self-energies $\Sigma_{11}$ and $\Sigma_{1 !}$ for pure spin states (and the energies $\left.\varepsilon_{\uparrow, \downarrow}\right)$ are real only on their Fermi surfaces, $p_{\uparrow, \downarrow}$. Away from the Fermi surfaces the single-particle states have a large attenuation, and the self-energies have imaginary parts which, close to the Fermi surface, are quadratic in 
the distance from this surface:

$$
\begin{aligned}
& \operatorname{Im} \Sigma_{\mid \uparrow}=\frac{1}{m^{*}} \sigma\left(p-p_{\uparrow}\right)^{2} \operatorname{sgn}\left(p-p_{\uparrow}\right), \\
& \operatorname{Im} \Sigma_{! \downarrow}=\frac{1}{m^{*}} \sigma\left(p-p_{!}\right)^{2} \operatorname{sgn}\left(p-p_{\downarrow}\right) .
\end{aligned}
$$

At low polarization, $\sigma$ is the same for up and down spins. In dilute systems, $\sigma=a^{2} p_{0}^{2} / \pi$ [7], where $a$ is the $s$-wave scattering length, and $p_{0}$ is the Fermi momentum without the polarization. This imaginary correction,

$i\left(\sigma / 2 T_{0}\right)\left[v_{0}^{2}\left(p_{\uparrow}-p_{\downarrow}\right)^{2}+2 \mathbf{k} \cdot \mathbf{v}_{0} v_{0}\left(p_{\uparrow}-p_{\downarrow}\right)+\left(\mathbf{k} \cdot \mathbf{v}_{0}\right)^{2}\right]$, modifies the dynamic part of the LSL transport equation.
The antisymmetric Landau interaction function $F^{(a)}\left(\mathrm{p}_{0}, \mathrm{p}_{0}^{\prime}\right)$ also has an imaginary part away from the Fermi surface. This correction is equivalent to adding $\left(i / 2 T_{0}\right) \gamma\left(\mathbf{p}_{0}, \mathbf{p}_{0}^{\prime}\right)\left[v_{0}\left(p_{\uparrow}-p_{\downarrow}\right)+\mathbf{k} \cdot \mathbf{v}_{0}\right]$ to the LSL value of $F^{(a)}\left(\mathrm{p}_{0}, \mathrm{p}_{0}^{\prime}\right)$. The microscopic definition of the function $\gamma\left(\mathrm{p}_{0}, \mathrm{p}_{0}^{\prime}\right)$ is more cumbersome than $\sigma$, and contains an integral equation in momentum derivatives of the exact irreducible vertex function in off-shell direction. In dilute systems $\gamma(\theta)=a^{2} p_{0}^{2} / 2 \pi \cos (\theta / 2)$. It is not an accident (see below) that the zeroth angular harmonic of $\gamma(\theta), \gamma_{0}$, is $a^{2} p_{0}^{2} / \pi$ and is equal to $\sigma$.

Finally, the microscopic equations [7] at $T=0$ and low polarization reduce to

$$
\begin{aligned}
\left\{\omega-2 \beta_{1} H-\mathbf{k} \cdot \mathbf{v}_{0}+i\left(\sigma / 2 T_{0}\right)[\right. & \left.\left.4 \beta_{1} H\left(\beta_{1} H+\mathbf{k} \cdot \mathbf{v}_{0}\right)+\left(\mathbf{k} \cdot \mathbf{v}_{0}\right)^{2}\right]\right\} \delta n \\
& -\left[2 \beta_{1} H+\mathbf{k} \cdot \mathbf{v}_{0}\right] \int\left[F^{(a)}\left(\mathbf{p}_{0}, \mathbf{p}_{0}^{\prime}\right)+i\left(2 \beta_{1} H+\mathbf{k} \cdot \mathbf{v}_{0}\right) \gamma\left(\mathbf{p}_{0}, \mathbf{p}_{0}^{\prime}\right) / 2 T_{0}\right] \delta n\left(\mathbf{p}_{0}^{\prime}\right) d p^{\prime} / 4 \pi=0,
\end{aligned}
$$

where $\delta \boldsymbol{n}$ is the distribution of transverse magnetization and $\beta_{1}=v_{0}\left(p_{\uparrow}-p_{\downarrow}\right) / 2 H$ determines the magnetic susceptibility. The real part of Eq. (2) is identical to the LSL equation.

Comparison with experiment requires the information at finite temperatures, when our equations should be modified. The logarithmic Fermi liquid singularity in the particle-hole channel at small energy transfer, which is responsible for the form of Landau-like equations, obtains a temperature cutoff and smears out. At $T \ll T_{0}$, this peak is still sharp, and the form of the equations is preserved. Another change concerns the temperaturedriven attenuation, i.e., a collision integral in the transport equation. Technically, one should use the finitetemperature (Keldysh or Kadanoff-Baym) diagrammatic techniques rather than the Dyson equation as in [7]. This can be done consistently for dilute Fermi gases [4], but is more difficult at higher density.

At low polarization and temperature $\tau_{\perp}(T, H)$ can be evaluated assuming that the inverse relaxation time $\tau_{\perp}{ }^{-1}$ has no strong singularity at $T, H \rightarrow 0$ and is small. This is confirmed by our calculations at $T=0$, standard calculations at $H=0$, and dilute-gas calculations [4] at arbitrary $T$ and $H$; this will be addressed in more detail elsewhere. The expansion of $\tau_{\perp}^{-1}$ starts from the terms of the order $\left(T / T_{0}\right)^{2}$ and $\left(\beta H / T_{0}\right)^{2}$ (the term $T H / T_{0}^{2}$ has a wrong time-reversal symmetry). With this accuracy, the temperature-driven and polarization-driven attenuations can be included into the transport equation independently from each other. The temperature-driven attenuation is equivalent to the following collision integral in the right hand side of Eq. (2):

$$
\begin{aligned}
I(\delta n)= & -\left(i / \tau_{\perp T}\right)\left(1+F_{1}^{(a)} / 3\right) \\
& \times\left[\delta n\left(\mathbf{p}_{0}\right)-\overline{\delta n\left(\mathbf{p}_{0}\right)}-3 \overline{\delta n\left(\mathbf{p}_{0}\right) \cos \phi} \cos \phi\right] .
\end{aligned}
$$

At low polarization $\tau_{\perp T}$ is equal to the usual longitudinal relaxation time [8]

$$
\tau_{\perp T}=\frac{32 \pi^{2}}{m^{3} T^{2}}\left\langle W(\theta, \phi) \frac{(1-\cos \theta)(1-\cos \phi)}{\cos \theta / 2}\right\rangle^{-1} C_{D},
$$

where $W(\theta, \phi)$ is the particles' scattering probability on the Fermi surface, $C_{D}$ is the Brooker-Sykes coefficient, and $\langle\cdots\rangle$ is the angular average. We will introduce an effective scattering length $a_{T}$ instead of the averaged scattering probability $W(\theta, \phi)$ so that $\tau_{\perp T}$ obtains the form similar to dilute systems, $\tau_{\perp T}^{-1}=8 \pi m a_{T}^{2} T^{2} / 3 C_{D}$.

The standard way to solve Eqs. (2) and (3) is to look for the solution in the form $\delta n\left(\mathrm{p}_{0}\right)=n_{0}+\mathrm{k} \cdot \mathrm{v}_{0} n_{1}$, and integrate the equation over angles. Since at $\mathbf{k}=0$ the eigenvalue is $\omega=\Omega_{0}\left(\Omega_{0}=2 \beta H\right.$ is the free Larmor frequency), the real and imaginary parts of Eqs. (3) and (4) at $\mathbf{k}=0$ give, after integration, two additional conditions: $\beta_{1}=\beta /\left(1+F_{0}^{(a)}\right)$ and $\sigma=\gamma_{0}$. The first condition is a usual expression for a magnetic susceptibility of a Fermi liquid. The second one is a similar relation for dissipative off-shell terms; it can be checked by comparison of imaginary parts of the mass operators and the vertex [7].

In the next step one should multiply Eqs. (2) and (3) by $\mathbf{k} \cdot \mathbf{v}_{0}$ and integrate again. These two integrations provide the eigenvalue of Eqs. (2) and (3),

$$
\begin{aligned}
& \omega=\Omega_{0}+\frac{1}{3} \frac{k^{2} v_{0}^{2}}{\Omega_{l}}\left(1+F_{0}^{(a)}\right)\left(1-\frac{i}{\Omega_{l} \tau_{\mathrm{eff}}}\right), \\
& \Omega_{l}=\frac{\left(F_{0}^{(a)}-F_{1}^{(a)} / 3\right) \Omega_{0}}{\left(1+F_{1}^{(a)} / 3\right)\left(1+F_{0}^{(a)}\right)} .
\end{aligned}
$$

The real part of Eq. (4) is identical to the spectrum of the Silin spin waves. The effective transverse relaxation time consists of the temperature-driven relaxation and the zero-temperature polarization-driven attenuation:

$$
\begin{aligned}
\tau_{\mathrm{eff}}^{-1} & =\tau_{\perp 0}^{-1}+\tau_{\perp T}^{-1}, \\
\tau_{\perp 0}^{-1} & =\frac{\Omega_{0}^{2}\left(\gamma_{0}-\gamma_{1} / 3\right)}{2 T_{0}\left(1+F_{0}^{(a)}\right)^{2}} \frac{1+2 F_{0}^{(a)}-F_{1}^{(a)} / 3}{\left(1+F_{1}^{(a)} / 3\right)^{2}} .
\end{aligned}
$$


The relaxation time $(5)$ has the necessary temperature dependence (1) with

$$
T_{a}=\frac{a_{H} C_{D}^{1 / 2} \Omega_{0}}{2^{1 / 2} \pi a_{T}\left(1+F_{0}^{(a)}\right)} \frac{\left(1+2 F_{0}^{(a)}-F_{1}^{(a)} / 3\right)^{1 / 2}}{1+F_{1}^{(a)} / 3},
$$

where we parametrized $\gamma_{0}-\gamma_{1} / 3$ as $\frac{4}{3} a_{H}^{2} p_{0}^{2} / \pi$. Effective lengths $a_{T}$ and $a_{H}$ can be expressed via the same vertex function, and are related to each other [e.g., $\gamma_{0}=\sigma$ is the attenuation of particles near the Fermi surface associated with the scattering of particles with the probability $W(\theta, \phi)$.] The explicit relation is unknown, except for dilute systems, and we have to consider $a_{T}$ and $a_{H}$ as independent parameters. In dilute systems $1 \gg F_{0}^{(a)} \gg F_{1}^{(a)}$, $a_{T}=a_{H}=a, C_{D} \approx 0.8$, and $T_{a}=\Omega_{0} C_{D}^{1 / 2} / 2^{1 / 2} \pi$. The difference from [4], $T_{a}=\Omega_{0} / 2 \pi$, is explained by a variational solution of the transport equation in [4]; our result is exact in the low-density low-polarization limit.

The transition temperature $T_{a}$ in ${ }^{3} \mathrm{He} \uparrow$ is $16.4 \pm 2.2$ $\mathrm{mK}$ [1] and is much higher than an extrapolation [1] $T_{a}^{(0)}=6.5 \mathrm{mK}$ from the variational dilute-gas results [4] with a heuristic substitute $\left.\Omega_{0} \rightarrow \Omega_{0} /\left(1+F_{0}{ }^{a}\right)\right) \approx 3.4 \Omega_{0}$ which was supposed to take care of the anomalously high magnetic susceptibility of ${ }^{3} \mathrm{He}$. Comparison with our equations gives a reasonable value of unknown parameters,

$$
\frac{a_{H}}{a_{T}} \frac{C_{D}^{1 / 2}\left(1+2 F_{0}^{(a)}-F_{1}^{(a)} / 3\right)^{1 / 2}}{1+F_{1}^{(a)} / 3} \approx 1.72 \pm 0.23 .
$$

The experiment [2] (the data still have a preliminary character) revealed the anomalous temperature dependence (1) of spin diffusion in ${ }^{3} \mathrm{He} \uparrow-{ }^{4} \mathrm{He}$ mixtures with $3.8 \%{ }^{3} \mathrm{He}\left(T_{0} \sim 290 \mathrm{mK}, \Omega_{0}=2 \beta H \sim 13.7 \mathrm{mK}, T \geq 13\right.$ $\mathrm{mK})$. This situation differs from pure ${ }^{3} \mathrm{He}$ because of the following anomaly [9]: The parameter $F_{0}^{(a)}-F_{1}^{(a)} / 3$ becomes zero at some critical concentration $x_{c}$ between $3 \%$ and $5 \%$, and the spectrum of Silin waves (4) has a singularity and should be reexamined. This has been done in [10] in the LSL approximation. According to [10], the spectrum $\omega$ near $x_{c}$ becomes linear in the wave vector $k$ even in a long-wave range instead of a standard $k^{2}$ dependence (4).

The zero-temperature attenuation modifies the results [10] and helps to preserve the $k^{2}$ form of the spectrum. Above we multiplied Eqs. (2) and (3) by $\mathbf{k} \cdot \mathbf{v}_{0}$ and integrated over angles. Usually, one neglects $\omega-\Omega_{0}$ in the resulting equation. This cannot be done at $\left|\chi-\chi_{c}\right| \ll 1$ [10]. Then, instead of (4), the spectrum reduces to

$$
\omega=\Omega_{0}+\frac{k^{2} v_{0}^{2}\left(1+F_{0}^{(a)}\right)^{2} / 3}{\Omega_{0} f\left(x_{c}-x\right)+i \Omega_{0}^{2}\left(\gamma_{0}-\gamma_{1} / 3\right) / 2 T_{0}\left(1+F_{0}^{(a)}\right)^{2}+i\left(1+F_{0}^{(a)}\right) / \tau_{\perp T}+\omega-\Omega_{0}}
$$

[wherever possible we substituted $F_{0}^{(a)}=F_{1}^{(a)} / 3$, neglected unimportant terms with $\gamma$, and parametrized the difference $F_{0}^{(a)}-F_{1}^{(a)} / 3$ in the denominator as $\left(1+F_{0}^{(a)}\right) f\left(x_{c}-x\right)$, where $x_{c}(T, H)=x_{c 0}+k_{H} \Omega_{0} / T_{0}+k_{T} T^{2} / T_{0}^{2}$, and $x_{c 0}$ is the critical concentration at $T=H=0$ ]. In the long-wave limit,

$$
k v_{0} \ll \frac{1}{1+F_{0}^{(a)}}\left|\Omega_{0} f\left(x_{c}(T, H)-x\right)+\frac{i \Omega_{0}^{2}\left(\gamma_{0}-\gamma_{1} / 3\right)}{2 T_{0}\left(1+F_{0}^{(a)}\right)^{2}}+\frac{i\left(1+F_{0}^{(a)}\right)}{\tau_{\perp T}}\right|,
$$

one can neglect $\omega-\Omega_{0}$ in the denominator (6), and the spectrum (6) remains quadratic. The spectrum is linear, as in [10], $\omega=\Omega_{0}+k v^{\prime} 0\left(1+F_{0}^{(a)}\right) / \sqrt{3}$, only for shorter waves. In contrast to [10], the zero-temperature attenuation makes the range (7) finite even at $x=x_{c}$ and $T=0$ :

$$
k v^{\prime}{ }_{0} \ll \Omega_{0}^{2}\left(\gamma_{0}-\gamma_{1} / 3\right) / 2 T_{0}\left(1+F_{0}^{(a)}\right)^{3} .
$$

The spectrum (6) near $x_{c}$ is very sensitive to the magnetic field. At the critical point $x_{c}$ the spectrum is purely diffusive, $\omega=\Omega_{0}-i D_{\mathrm{eff}} k^{2}$,

$$
D_{\mathrm{eff}}=\frac{v_{0}^{2}\left(1+F_{0}^{(a)}\right) \tau_{\perp T} / 3}{1+\tau_{\perp T} \Omega_{0}^{2}\left(\gamma_{0}-\gamma_{1} / 3\right) / 2 T_{0}\left(1+F_{0}^{(a)}\right)^{3}} .
$$

Away from $x_{c}$ the spectrum acquires a spin rotation part and recovers the form (5).

In experiment [2] the spin rotation was virtually unobservable, and the spin diffusion coefficient is equal to $D_{\text {eff }}$ above. With the same parametrization for $\tau_{\perp T}$ and $\gamma_{0}-\gamma_{1} / 3$, the temperature $T_{a}=\left(\Omega_{0} / \pi\right)\left(C_{D} / 2\right)^{1 / 2}\left(a_{H} /\right.$ $\left.a_{T}\right)\left(1+F_{0}^{(a)}\right)^{-3 / 2}$. Since $\Omega_{0} \approx 14 \mathrm{mK}$, the data [2] $T_{a}$ $\approx 14 \mathrm{mK}$ mean that $\left(a_{H} / a_{T}\right) C_{D}^{1 / 2}\left(1+F_{0}^{(a)}\right)^{-3 / 2} \approx 4.5$.
This value seems to be feasible though relatively large.

The large value of $a_{H} / a_{T}\left(1+F_{0}^{(a)}\right)^{3 / 2}$ at $x=3.8 \%$ is important especially because at this concentration $F_{0}^{(a)}$ $-F_{1}^{(a)} \approx 0$. Both facts signal a strong deviation from a single extrapolation of a low concentration behavior when $F_{0}^{(a)}$ is positive and increases as $x^{1 / 3}$, while $F_{1}^{(a)}$ is much smaller and is proportional to $x^{2 / 3}$. The inevitable conclusion is that at $x>(2-3) \%$ the harmonic $F_{0}^{(a)}$ in helium mixtures is suppressed with respect to its low concentration value. This, in turn, means that the effective attractive interaction at this concentration is much weaker than its extrapolation from low concentrations with the $s$-wave scattering length $a \approx-1 \AA$ [11]. If this is true, all current attempts to observe ${ }^{3} \mathrm{He}$ superfluidity in ${ }^{3} \mathrm{He}$ ${ }^{4} \mathrm{He}$ mixtures are hopeless.

We are grateful to D. Candela and J. Owers-Bradley for numerous discussions of experimental situation. The work was supported by NSF DMR-9100197.

\footnotetext{
*Present address: Department of Physics, University of
} 
Wisconsin, Madison, WI 53706.

[1] L.-J. Wei, N. Kalenchofsky, and D. Candela, Phys. Rev. Lett. 71, 879 (1993).

[2] J. Owers-Bradley, A. Child, and R. M. Bowley, in Proceedings of the Twentieth International Conference on Low Temperature Superconductors, Eugene, 1993 (to be published).

[3] A. E. Meyerovich, Phys. Lett. 107A, 177 (1985); see also the review in Helium Three, edited by W. P. Halperin and L. P. Pitaevskii (North-Holland, Amsterdam, 1990), pp. 757-879, and references therein.

[4] J. W. Jeon and W. J. Mullin, J. Phys. (Paris) 49, 1691 (1988); Phys. Rev. Lett. 62, 2691 (1989); J. Low Temp. Phys. 88, 433 (1992).

[5] V. P. Silin, Sov. Phys. JETP 6, 945 (1957); A. J. Leggett,
J. Phys. C 3, 448 (1970); P. M. Platzman and P. A. Wolf, Waves and Interaction in Solid State Plasmas, Solid State Physics Suppl. 13 (Academic, New York, 1.973).

[6] A. E. Meyerovich, Physica (Amsterdam) 169B, 183 (1991).

[7] A. E. Meyerovich and K. A. Musaelian, J. Low Temp. Phys. 89, 781 (1992); J. Low Temp. Phys. (to be published).

[8] D. Hone, Phys. Rev. 121, 669 (1961); G. A. Brooker and J. Sykes, Phys. Rev. Lett. 21, 279 (1968).

[9] H. Ishimoto et al., Phys. Rev. Lett. 59, 904 (1987).

[10] K. S. Bedell, Phys. Rev. Lett. 62, 167 (1989).

[11] J. R. Owers-Bradley et al., J. Low Temp. Phys. 88, 221 (1992). 